\title{
A Resolution to the Vacuum Catastrophe
}

\author{
Siamak Tafazoli*
}

\begin{abstract}
This paper presents a theoretical estimate for the vacuum energy density which turns out to be near zero and thus much more palatable than an infinite or a very large theoretical value obtained by imposing an ultraviolet frequency cut-off. This result helps address the "vacuum catastrophe" and the "cosmological constant problem".
\end{abstract}

The vacuum energy density (or zero-point energy density, i.e. energy $E$ per volume $V$ ) of a free quantum field is given by [1],

$$
\hat{\rho}_{\text {vac }}=\frac{(-1)^{2 j}(2 j+1)}{2} \int_{-\infty}^{\infty} \frac{1}{(2 \pi)^{3}} \sqrt{m^{2}+\mathbf{k}^{2}} d^{3} k=\frac{(-1)^{2 j}(2 j+1)}{2} \int_{0}^{\infty} \frac{4 \pi}{(2 \pi)^{3}} \sqrt{m^{2}+k^{2}} k^{2} d k
$$

in natural units where the reduced Planck constant $\hbar=$ the speed of light in vacuum $c=1$. Note that $m$ is the particle mass associated with a specific field, $j$ is the spin, $\mathbf{k}$ is the wave vector and $k$ is the wave number. For a massless field (i.e. photon and gluon, with $m=0$ and $j=1$ ), we can further simplify (1) to

$$
\hat{\rho}_{v a c}=\frac{(-1)^{2 j}(2 j+1)}{2} \frac{1}{2 \pi^{2}} \int_{0}^{\infty} k^{3} d k
$$

For massive fields (i.e. electron and quarks $\left(j=\frac{1}{2}\right), \mathrm{W} \& \mathrm{Z}(j=1)$ and $\operatorname{Higgs}(j=0)$, and with $m \neq 0$ ), equation (2) provides a good approximation which gets better for smaller $m$.

At first glance, the integral in (2) looks highly divergent, however using the results obtained in [2], a value for this integral can be calculated to be $\frac{(-1)^{3+1}}{(3+1)(3+2)}=\frac{1}{20}$ and hence the expectation value of the vacuum energy density does not diverge and is nearly zero. The vacuum energy density contributions from all the fields (note that the lepton (e.g. electron) and quark fields have a negative vacuum energy density contribution) can then be summed up which results in a positive near zero value, given in metric units by multiplying (2) with values of $\hbar$ and $c$ :

$$
\hat{\rho}_{v a c} \approx 2.4 \times 10^{-28} \mathrm{~J} / \mathrm{m}^{3}
$$

This value agrees with the large scale cosmological observations which set an upper bound of approximately $10^{-11} \mathrm{~J} / \mathrm{m}^{3}$ on the vacuum energy density [3]. It also agrees with the predictions of current cosmological models and all observational data to date which essentially has found that vacuum has very little energy content leading to a vanishing cosmological constant. This result helps address the "vacuum catastrophe" and the "cosmological constant problem".

*C Siamak Tafazoli, email: mak.tafazoli@gmail.com 


\section{References}

[1] R. D. Klauber, "The Student Friendly Quantum Field Theory", 2013.

[2] F. Aghili and S. Tafazoli, "Analytical Solution to Improper Integral of Divergent Power Functions Using The Riemann Zeta Function", 2018.

[3] S. M. Carroll, "The Cosmological Constant", 2000. 